\title{
Malignant Phyllodes Tumor of the Breast in a 26- year-old Woman
}

\author{
Nga T. Nguyen ${ }^{1}$, Lynsey M. Maciolek ${ }^{1}$, Suimin Qiu ${ }^{2}$, Sarfaraz Sadruddin ${ }^{1}$, Quan D. Nguyen ${ }^{1}$ \\ 1. Radiology, University of Texas Medical Branch, Galveston, USA 2. Pathology, University of Texas Medical Branch, \\ Galveston, USA
}

Corresponding author: Quan D. Nguyen, qunguyen@utmb.edu

\begin{abstract}
Breast cancer is the most commonly diagnosed cancer and the second leading cause of cancer death in women. Early detection, accurate diagnosis and proper treatment are important prognostic factors due to the wide variety of breast cancer subtypes. This becomes of particular importance with rare breast tumors, which are difficult to diagnose due to their varying presentations. Malignant phyllodes tumor (PT) is one of the rare breast tumors that is difficult to diagnose. First reported in 1838, PT of the breast accounts for less than $1 \%$ of all breast masses. Based on histological features, PT is categorized on a spectrum of benign to malignant tumors. High-grade PTs are commonly seen in older patients but can also occur in young patients, as reported in this case of a 26 -year-old female. Failure to detect the malignancy early and initiate appropriate treatment can lead to widespread metastasis and poor outcomes.
\end{abstract}

Categories: Pathology, Radiology, Oncology

Keywords: li-fraumeni syndrome, malignant phyllodes tumor, myofibrosarcoma, fibroadenoma, phyllodes tumor, borderline phyllodes tumor, breast carcinoma, leaf-like projections, breast sarcoma, p53 gene

\section{Introduction}

In the past few decades, with easier access to high-quality prevention, early detection and advanced treatment, breast cancer mortality rates have declined significantly, dropping 40\% from 1989 to 2017 [1]. While treatments for common types of breast cancer have been extensively studied and made available to patients, the pathogenesis and course of treatment for rarer breast tumors have not been well established. Phyllodes tumors (PTs) of the breast belong to one of those rare entities.

Received 12/02/2019

Review began 12/06/2019 Review ended 01/06/2020 Published 01/07/2020

\section{() Copyright 2020}

Nguyen et al. This is an open access article distributed under the terms of the Creative Commons Attribution License CC-BY 3.0., which permits unrestricted use, distribution, and reproduction in any medium, provided the original author and source are credited.
PTs of the breast account for $0.3 \%$ to $1 \%$ of all breast tumors [2]. They are most commonly diagnosed in the elderly population. Unlike the more common type of carcinoma caused by neoplastic epithelial cells, these tumors originate from the connective tissue, stroma, which provides the supportive framework for the lobules, ducts, blood vessels and lymph. PTs of the breast are classified by the World Health Organization into benign, borderline and malignant with varying presentations, prognoses and courses of treatment [3]. While malignant PTs can metastasize with poor outcomes and high rates of recurrence, benign and borderline tumors present with good prognosis and lower rates of recurrence [2]. The classification of PT is established based on specific histological criteria, including cellular atypia, number of mitoses, nuclear uniformity, stromal cellularity and border infiltration. Nevertheless, the differentiation between PT subtypes and the respective differential diagnoses is not without controversy.

Because of the challenges in providing an accurate diagnosis and the predilection of PT in the older population, malignant PTs in the younger generations can be overlooked. Different subtypes of PT have different courses of treatment; therefore, underdiagnosis or overdiagnosis can lead to inadequate or unnecessary interventions. Cases of malignant PTs in young patients have been reported, but it is very rare for a patient to be diagnosed with both borderline and malignant PTs. This paper details the presentation of malignant PT of the right breast in a very young woman with a past medical history of sarcoma and borderline PT of the left breast. Highlights of imaging features, pathohistological findings, basic histochemistry, differential diagnoses and management are also presented.

\section{Case Presentation}

A 26-year-old woman presented with an enlarging tender mass in the right breast. Her past medical history was significant for multiple breast tumors. At age 19 years, a sarcoma of the left breast arising from a tubular adenoma was discovered. At that time, she underwent a segmental mastectomy. At age 20 years, she was diagnosed with a borderline PT of the same breast, treated with a nipple sparing mastectomy of the left breast. The patient's family history was significant for breast cancer in two maternal aunts. On physical examination of the right breast, a moveable mass at three o'clock at a distance of $2 \mathrm{~cm}$ from the nipple was palpated. A targeted ultrasound of the right breast revealed a hypoechoic mass at the site of the clinically palpable mass with an abrupt interface, circumscribed margins, a combined pattern of posterior acoustics and an oval/lobulated shape, as seen in Figure 1. 


\section{Cureus}

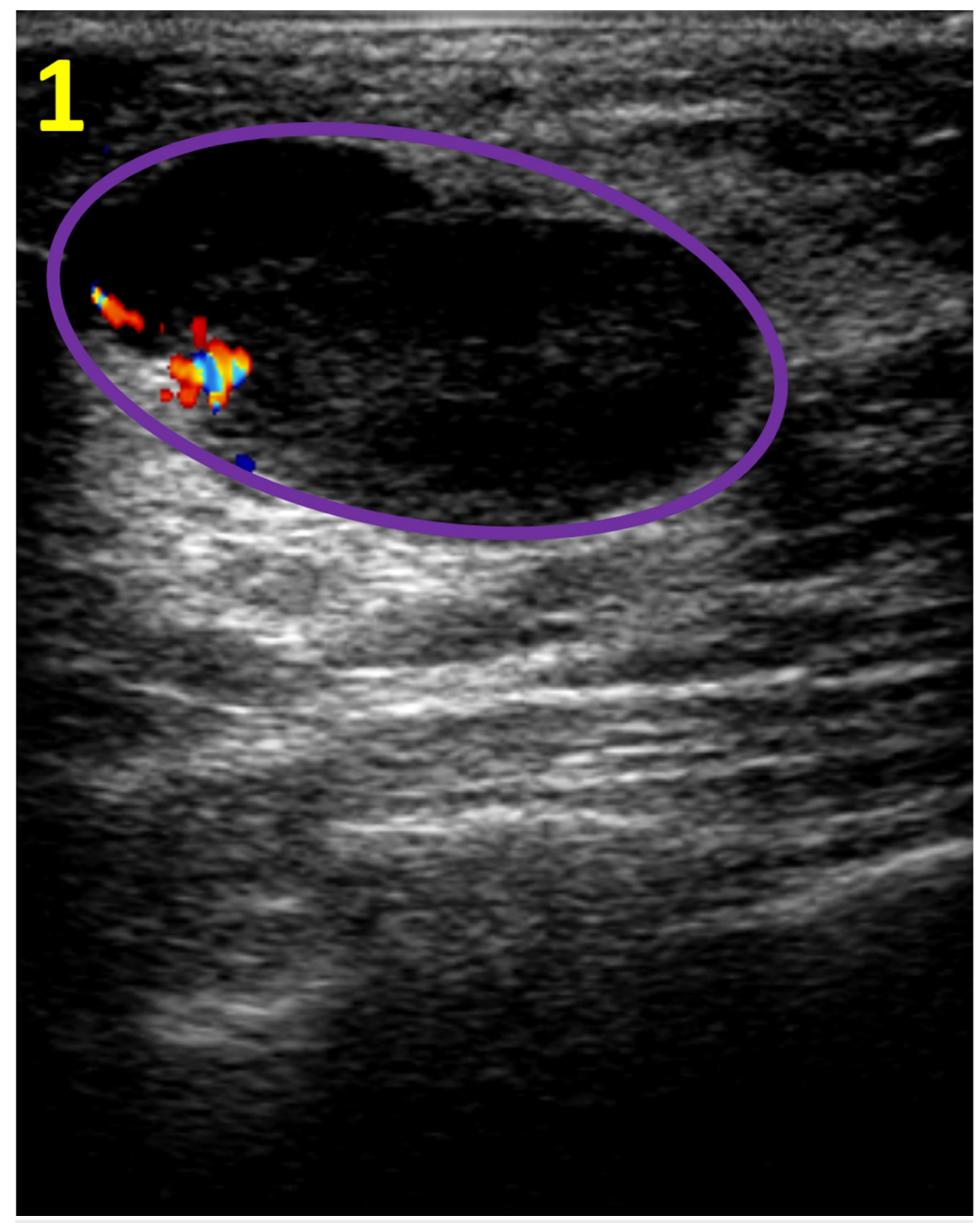

\section{FIGURE 1: Targeted Ultrasound of the Right Breast}

Targeted ultrasound of the right breast demonstrates a solid mass (purple circle) measuring $26 \times 20 \times 16 \mathrm{~mm}$ at the site of the clinically palpable mass at three o'clock at a distance of $2 \mathrm{~cm}$ from the nipple. The characteristics of the finding include a hypoechoic pattern, an abrupt interface, circumscribed margins, an orientation that is parallel to the skin line, a combined pattern of posterior acoustics and an oval shape.

The right axilla demonstrated no lymphadenopathy. Because of her significant past medical history, current presentation of an enlarging right breast mass and the sheer size of the tumor, primary breast malignancy was raised as a concern. Subsequently, an ultrasound-guided core needle biopsy of the right breast was performed for a more accurate tissue diagnosis (Figures 2, 3). 


\section{Cureus}



FIGURE 2: Ultrasound-guided Core Needle Biopsy of the Right Breast

The hyperechoic biopsy needle (orange asterisk) is visualized within the right breast mass. 


\section{Cureus}

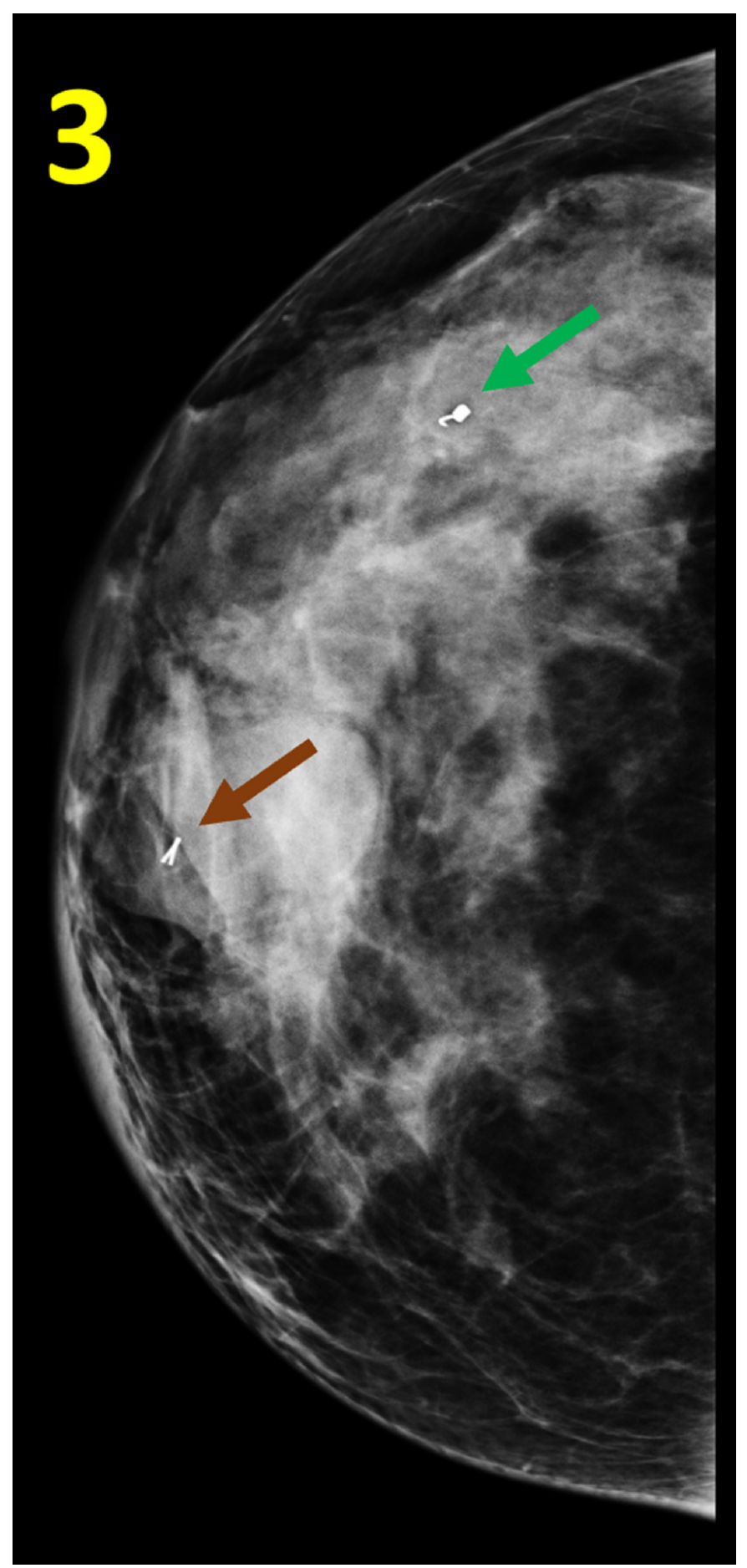

FIGURE 3: Post-biopsy Mammogram of the Right Breast in Cranialcaudal View

A micromarker (RIBBON) was placed at the site of the core biopsy. The clip (RIBBON) (brown arrow) was present within the mass. An additional clip (COIL) (green arrow) was noted in the upper outer quadrant of the right breast. The patient could not provide any history regarding this biopsy. 


\section{Cureus}

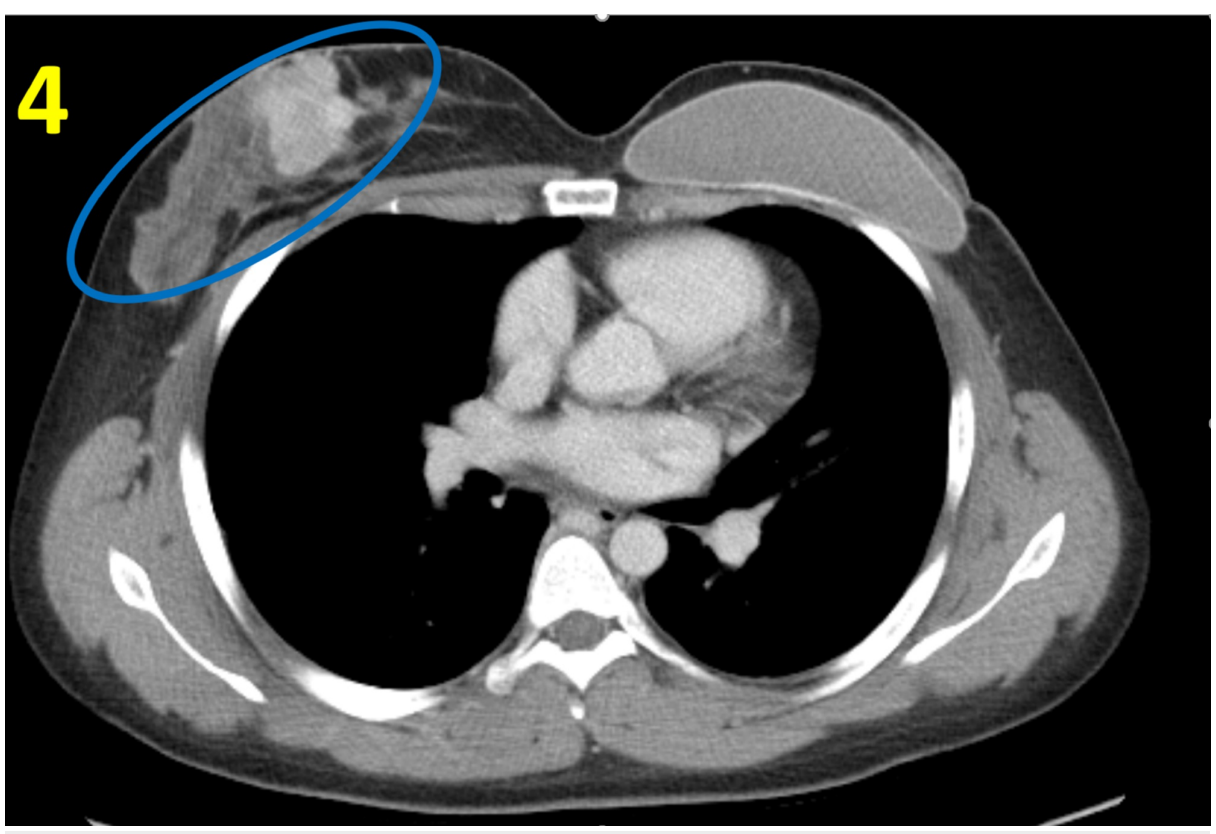

FIGURE 4: Computed Tomography Scan of the Thorax

An enhancing mass (blue circle) was seen in the right breast, in concordance with the right breast malignancy. No evidence of metastatic disease was found.

This scan indeed ruled out metastatic disease in this patient. The patient eventually had a simple mastectomy of the right breast. Histology of the mastectomy tissue demonstrated hypercellularity with malignant stromal cells and increased mitotic activity (30 mitoses per 10 high-power fields) (Figure 5).

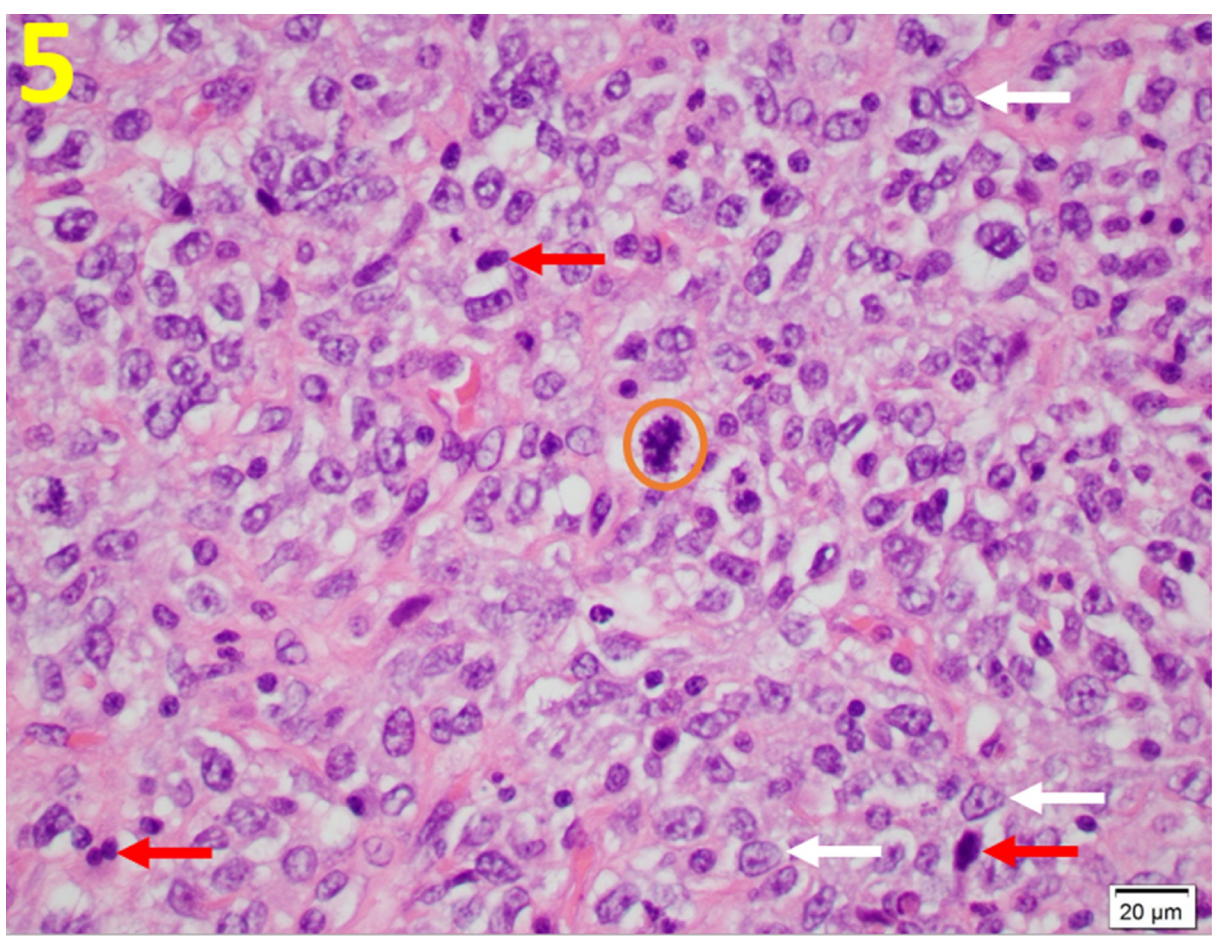

FIGURE 5: Nuclear Pleomorphism in Malignant Phyllodes Tumors

The stroma cells show marked atypia with numerous mitoses (orange circle), prominent nucleoli (white arrows) and high nuclear-to-cytoplasmic ratio (red arrows). This is demonstrated on a hematoxylin and eosin stain at a magnification of $400 x$.

Benign epithelium and periductal condensation of atypical stromal cells was also present (Figure 6). 


\section{Cureus}

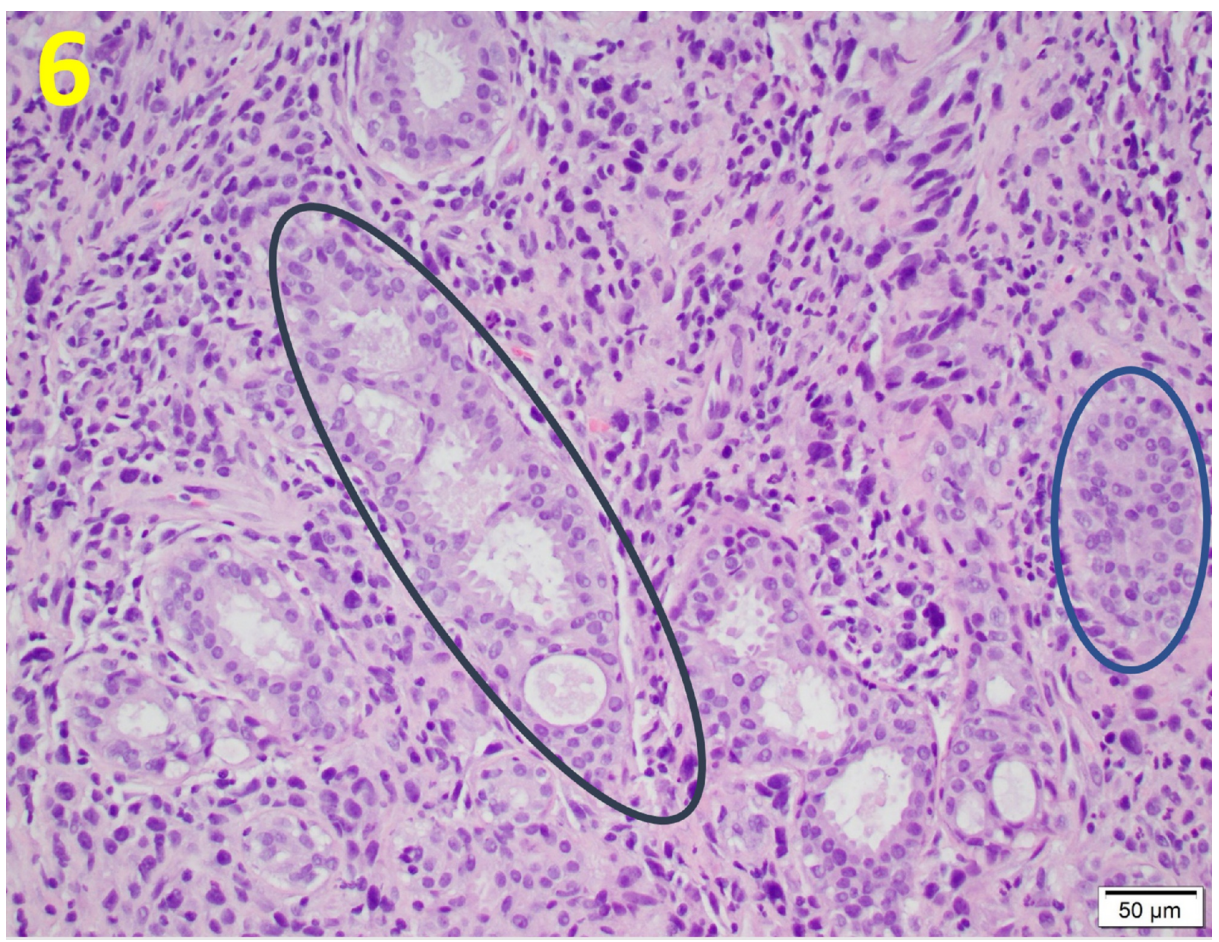

\section{FIGURE 6: Malignant Stromal Cell Proliferation}

The presence of benign epithelium and periductal condensation of atypical stromal cells (black circle) is typical of malignant phyllodes tumors. There is also evidence of multinucleated giant cells (blue circle). This is demonstrated on a hematoxylin and eosin stain at a magnification of 200x.

The tissue showed multinucleated giant cells, which are commonly seen in malignant PTs. Additionally, a leaf-like architecture and hypercellular stroma that resembled fibroblasts and myofibroblasts were noted (Figure 7).



FIGURE 7: Leaf-like Projection of Phyllodes Tumors

Leaf-like projection of the epithelial lining (shape formed by the blue arrows) into cystic spaces is a classic 


\section{Cureus}

histological finding in phyllodes tumor. This is demonstrated on a hematoxylin and eosin stain at a magnification of $100 x$.

Immunohistochemical stains were diffusely positive for cluster of differentiation 34 (CD34) and vimentin and focally positive for caldesmon (Figures 8, 9).



FIGURE 8: CD34 Positivity in Phyllodes Tumor

CD34 positively stained spindle cells (yellow brown) are common in malignant phyllodes tumors. This is demonstrated at a magnification of $200 x$.

CD34: cluster of differentiation 34 


\section{Cureus}



\section{FIGURE 9: Focal Positivity for Caldesmon Stain in Phyllodes Tumor}

Caldesmon positivity (yellow brown) is a common histochemical finding in malignant phyllodes tumors. This is demonstrated at a magnification of $200 x$.

The tissue was negative for epithelial membrane antigen, cytokeratin AE1/3, cytokeratin 7, desmin, p63 and melan-A. The morphological findings and immunohistochemical staining profile were consistent with the diagnosis of primary malignant PTs of the right breast. Beyond this, metastasis from the previously diagnosed PT from the left breast was ruled out.

The patient received one round of adjuvant radiation to the breast and was monitored for recurrence. At two years of oncologic surveillance, the patient did not show any signs of active metastasis.

\section{Discussion}

Among the three subtypes of PT, malignant PT is the rarest entity which accounts for approximately $10 \%$ to $30 \%$ of all PTs [4]. Although malignant PT typically presents in the fourth to sixth decades of life, it can also occur in younger women. In a study completed by Abusalem et al., it was estimated that one out of 26 PTs was deemed to be malignant in patients $20-29$ years of age $[5,6]$. Grossly, malignant PT has varying clinical presentations in size, growth pattern and changes in the overlying skin [7]. Microscopically, malignant PTs are characterized by the leaf-like protrusions and elongated cleft-like spaces that contain papillary projections of stromal overgrowth with epithelial lining [3]. Histologically, malignant PT is differentiated from other subtypes by prominent stromal overgrowth and atypia, infiltrative margins and high mitotic rate (more than 10 mitoses per 10 high-power fields) [6,8]. Other sarcomatous features including

chondrosarcoma, liposarcoma and rhabdosarcoma can also be present [9]. Tumor markers for malignant PT are variable. Immunochemistry of malignant PT is often negative for p63 and cytokeratins [10]. However, studies have shown that these two markers can be positive in $57 \%$ and $21 \%$, respectively for malignant PTs [11]. Staining positive for CD34 is another useful diagnostic tool but only seen in $37 \%$ to $57 \%$ of malignant PTs [12]. Therefore, these markers need to be carefully assessed in combination with histological features to differentiate benign from malignant tumors.

An important pathology to consider in the differential is myofibrosarcoma (MFS), which can also stain positive for CD34 and demonstrate stromal overgrowth with cellular atypia on histology. However, histopathology findings in MFS typically include medium-to-large spindle cells grouped in large and irregular fascicles, which are separated by broad strands of hyalinized collagen fibers, fat cells or myxoid matrix [13]. Additionally, the leaf-like epithelial component seen in malignant PTs is not present in MFS.

Beyond the histological components of malignant PT, the appearance of malignant PT on sonographic imaging as a smooth, poly-lobulated mass resembles a fibroadenoma (FA) [14]. More specifically, malignant masses can share common features with complex FA which present variably on ultrasound, ranging from a 
well-circumscribed hypoechoic mass to a heterogenous mass with features of malignancy, including illdefined margin, lobulation, and microcalcification [14]. The presence of intralesional clefts and cystic spaces on ultrasound may favor PTs [14]. However, these characteristics have not been proven reliably useful for differentiation. On mammography, PTs are round or oval with either well- or ill-defined margins, which are very non-specific and make distinguishing PT from other neoplasms difficult. Because of the minimal values of mammography and sonography in providing an accurate diagnosis, magnetic resonance imaging (MRI) has been suggested as a useful diagnostic tool. The presence of a heterogenous inner structure and nonenhancing septations on MRI may indicate a diagnosis of PT more often than histologically proven FA [15]. Additionally, slit-like patterns in enhanced images and signal changes from T2-weighted to enhanced images are common features in PT. Breast imaging-reporting and data system (BI-RADS) categories are also strongly associated with histological grade of PTs. BI-RADS category 4a on mammography, category 3 on ultrasound and category $4 \mathrm{~b}$ on MRI are most commonly seen in high-grade PTs [14]. BI-RADS category $\geqslant 4 \mathrm{a}$ is considered to be suspicious for malignancy and, hence, warrants further intervention [14].

The aforementioned features are critical for radiologists to be aware of, as FA is the most common breast tumor in adolescent and young females. Therefore, malignant PTs may oftentimes be overlooked as a potential differential diagnosis. Histologically, FA share the fibroblastic and stromal overgrowth features with malignant PT, but they have rare mitotic figures in both glandular and stromal components, which are common in malignant PT. Furthermore, stromal overgrowth in FA is generally more uniform and without cellular atypia. Malignant PTs may also have clusters of multinucleated giant cells, which are typically not present in FA.

Although not required for diagnosis, malignant PTs have been shown to be highly associated with mutations in mediator complex subunit 12 in addition to several oncogenes and tumor suppressors, including p53, retinoblastoma, neurofibromin 1 and epidermal growth factor receptor [16,17]. Cytogenic studies have also demonstrated a strong correlation between borderline/malignant PT and 1q gain, and 9p21 deletion associated with loss of p16-Ink4a expression [17,18]. This patient's past medical history of multiple breast tumors, including a sarcoma and a borderline PT at a very young age, raises suspicion for a genetic component in the development of this rare malignant PT. Specifically, a past medical history of breast sarcoma at age 19 years is concerning for Li-Fraumeni syndrome, which is a rare genetic condition caused by p53 mutations. Li-Fraumeni syndrome has also been associated with an increased risk of developing PT [19].

The management of most cases of malignant PTs recommended by National Comprehensive Cancer Network guidelines includes complete surgical resection with negative margins of at least $1 \mathrm{~cm}$. Mastectomy is generally not recommended because of concern for poor cosmetic outcomes, unless positive margins cannot be achieved or if the tumor is too large to be completely excised. Adjuvant radiation therapy is controversial in terms of preventing tumor recurrence. Radiation was found to prevent recurrence of borderline and malignant PTs following surgical interventions (breast-conserving surgery and mastectomy) but was found to have no impact on disease-free survival [20]. Because our patient had a strong family history of breast cancer and also a past medical history of multiple high-grade breast tumors, it was determined that a partial mastectomy of the right breast would be the appropriate course of treatment, followed by adjuvant radiation therapy and annual surveillance mammography.

\section{Conclusions}

Although most PTs are benign, early detection of the malignant subtype of PT is important. A past medical history of multiple breast tumors and a significant family history raise suspicion for a genetic component in this rare entity. Current literature suggests chromosome imbalances and gene deletions as possible causes of malignant PT, but the exact etiology remains unknown. Future research focused on the hereditary aspect of malignant PT can shed light on the etiopathogenesis of this rare type of tumor and its possible relationship with other disease processes.

\section{Additional Information \\ Disclosures}

Human subjects: Consent was obtained by all participants in this study. Conflicts of interest: In compliance with the ICMJE uniform disclosure form, all authors declare the following: Payment/services info: All authors have declared that no financial support was received from any organization for the submitted work. Financial relationships: All authors have declared that they have no financial relationships at present or within the previous three years with any organizations that might have an interest in the submitted work. Other relationships: All authors have declared that there are no other relationships or activities that could appear to have influenced the submitted work.

\section{References}

1. Siegel RL, Miller KD, Jemal A: Cancer statistics, 2019. CA Cancer J Clin. 2019, 69:7-34. 10.3322/caac.21551

2. Zhang Y, Kleer CG: Phyllodes tumor of the breast: histopathologic features, differential diagnosis, and molecular/genetic updates. Arch Pathol Lab Med. 2016, 140:665-671. 10.5858/arpa.2016-0042-RA

3. The International Agency for Research on Cancer: WHO Classification of Tumours: Pathology and Genetics 
of Tumours of the Breast and Female Genital Organs. Tavassoli FA, Devilee P (ed): IARC Press, Lyon; 2003.

4. Roberts N and Runk DM: Aggressive malignant phyllodes tumor. Int J Surg Case Rep. 2015, 8:161-165. 10.1016/j.ijscr.2014.12.041

5. Abusalem OT, Al-Masri A: Phyllodes tumors of the breast. Mater Sociomed. 2011, 23:200-205. 10.5455/msm.2011.23.200-205

6. Reinfuss M, Mituś J, Duda K, Stelmach A, Ryś J, Smolak K: The treatment and prognosis of patients with phyllodes tumor of the breast: an analysis of 170 cases. Cancer. 1996, 77:910-916. 10.1002/(sici)10970142(19960301)77:5<910::aid-cncr16>3.0.c0;2-6

7. Calhoun KE, Allison KH, Kim JN, Rahbar H, Anderson BO: Phyllodes Tumors. Diseases of the breast. Harris JR, Lippman ME, Morrow M, Osborne CK (ed): Lippincott williams and wilkins, Philadelphia; 2010. 4:781792 .

8. Norris HJ, Taylor HB: Relationship of histologic features to behavior of cystosarcoma phyllodes. analysis of ninety-four cases. Cancer. 1967, 20:2090-2099. 10.1002/1097-0142(196712)20:12<2090::aidcncr2820201206>3.0.co;2-1

9. Guerrero MA, Ballard BR, Grau AM: Malignant phyllodes tumor of the breast: review of the literature and case report of stromal overgrowth. Surg Oncol. 2003, 12:27-37. 10.1016/s0960-7404(03)00005-7

10. Chia Y, Thike AA, Cheok PY, Yong-Zheng Chong L, Man-Kit Tse G, Tan PH: Stromal keratin expression in phyllodes tumours of the breast: a comparison with other spindle cell breast lesions. J Clin Pathol. 2012, 65:339-347. 10.1136/jclinpath-2011-200377

11. Cimino-Mathews A, Sharma R, Illei PB, Vang R, Argani P: A subset of malignant phyllodes tumors express p63 and p40: a diagnostic pitfall in breast core needle biopsies. Am J Surg Pathol. 2014, 38:1689-1696. 10.1097/PAS.0000000000000301

12. Moore T, Lee AH: Expression of cd34 and bcl-2 in phyllodes tumours, fibroadenomas and spindle cell lesions of the breast. Histopathology. 2001, 38:62-67. 10.1046/j.1365-2559.2001.01053.x

13. Taccagni G, Rovere E, Masullo M, Christensen L, Eyden B: Myofibrosarcoma of the breast: review of the literature on myofibroblastic tumors and criteria for defining myofibroblastic differentiation. Am J Surg Pathol. 1997, 21:489-496. 10.1097/00000478-199704000-00017

14. Tan H, Zhang S, Liu H, et al.: Imaging findings in phyllodes tumors of the breast. Eur J Radiol. 2012, 81:e62e69. 10.1016/j.ejrad.2011.01.085

15. Wurdinger S, Herzog AB, Fischer DR, et al.: Differentiation of phyllodes breast tumors from fibroadenomas on MRI. AJR Am J Roentgenol. 2005, 185:1317-1321. 10.2214/ajr.04.1620

16. Tan J, Ong CK, Lim WK, et al.: Genomic landscapes of breast fibroepithelial tumors . Nat Genet. 2015, 47:1341-1345. 10.1038/ng.3409

17. Cani AK, Hovelson DH, McDaniel AS, et al.: Next-gen sequencing exposes frequent med12 mutations and actionable therapeutic targets in phyllodes tumors. Mol Cancer Res. 2015, 13:613-619. 10.1158/15417786.MCR-14-0578

18. Jones AM, Mitter R, Springall R, et al.: A comprehensive genetic profile of phyllodes tumours of the breast detects important mutations, intra-tumoral genetic heterogeneity and new genetic changes on recurrence. J Pathol. 2008, 214:533-544. 10.1002/path.2320

19. Birch JM, Alston RD, McNally RJ, et al.: Relative frequency and morphology of cancers in carriers of germline tp53 mutations. Oncogene. 2001, 20:4621-4628. 10.1038/sj.onc.1204621

20. Zeng S, Zhang X, Yang D, Wang X, Ren G: Effects of adjuvant radiotherapy on borderline and malignant phyllodes tumors: a systematic review and meta-analysis. Mol Clin Oncol. 2015, 3:663-671. 10.3892/mco.2015.503 